\title{
Capacitação de Profissionais do Programa de SaÚde da Família em Estratégias de DiAGNÓSTICO E INTERVENÇ̃̃es BREVES PARA O USO PROBLEMÁTICO DE ÁlCOOL \\ Clarissa M. Corradi-Webster ${ }^{1}$; Elaine C. Minto ${ }^{1}$; Fabrízia Maria C. de Aquino ${ }^{1}$; Flávia Abade ${ }^{2}$; Lincoln Luiz Yosetake ${ }^{3}$; Ricardo Gorayeb ${ }^{4}$; Milton Roberto Laprega ${ }^{5}$; Erikson Felipe Furtado ${ }^{6}$
}

Visando a prevenção do uso problemático de álcool, a OMS desenvolveu estratégias de intervenções breves (EDIBs), disseminadas no Brasil, desde 2002, pelo PAI-PAD/OMS da FMRP-USP. Este estudo objetivou avaliar benefícios do treinamento em EDIBs para equipes do PSF, com uso de grupo focal. O perfil dos grupos refletiu a distribuição observada no treinamento ( $60 \%$ de agentes, $85 \%$ mulheres, e $51 \%$ de adultos abaixo de 35 anos). Identificaram-se benefícios relacionados aos objetivos cognitivos e atitudinais: questionamento de estereótipos; atenção para investigação sistemática; e dependência de álcool vista como condição de enfermidade. Romper estereótipos e compreender o padrão de consumo foi identificado pelos profissionais como benefícios do treinamento, tendo favorecido a implementação das EDIBs.

Palavras-chave: Atenção primária; medicina de família; uso problemático de álcool; intervenção breve; treinamento de profissionais de saúde; estudo qualitativo; grupo focal

\section{CAlificaCión de Profesionales del PROGRAMa de SALUd de la FAMILIA EN ESTRATEgIAS

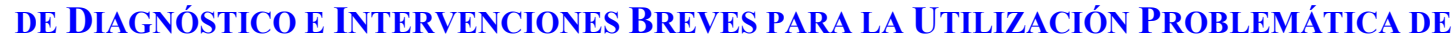 ALCOHOL}

Con una visón preventiva de la utilización problemática de alcohol, la OMS desarrolló estrategias de intervenciones breves (EDIBs), difundidas en Brasil desde 2002, por PAI-PAD/OMS de FMRP-USP. Este estudio pretendió evaluar los beneficios del entrenamiento en EDIBs para equipos de PSF, con utilización de grupos focales. El perfil de los grupos observados en el entrenamiento fue: $60 \%$ de agentes, $85 \%$ mujeres, y $51 \%$ de adultos menores de 35 años. Los beneficios identificados se relacionaron con el conocimiento y cambios de actitud: cuestionamiento de estereotipos; atención para la evaluación sistemática; y dependencia al alcohol como una condición de enfermedad. Romper estereotipos e incluir el patrón de consumo fueron identificados por los profesionales como los beneficios del entrenamiento, favoreciendo la utilización de de las EDIBs.

Palabras claves: Atención primaria; medicina de familia; utilización problemática de alcohol; intervención breve; formación de profesionales de salud; estudio cualitativo; grupo focal.

\section{Qualification of Family Health Program Professionals in Brief Diagnosis and INTERVENTION STRATEgIES FOR Problematic AlCOHOL USE}

With a view to preventing problematic alcohol use, the WHO developed brief intervention strategies (BDIS), disseminated in Brazil, since 2002, by PAI-PAD/WHO of the FMRP-USP. This study aimed to evaluate the benefits of BDIS training with the use of focal groups for FHP teams. The group profile reflected the distribution observed during training $(60 \%$ agents, $85 \%$ women, and $51 \%$ adults under 35$)$. The identified benefits were related to the cognitive and behavior change objectives: questioning of stereotypes; attention to systematic assessment; and alcohol dependence seen as a disease. The professionals identified breaking stereotypes and understanding drinking patterns as training benefits, which favored the implementation of the BDIS.

Key words: Primary care; family medicine; problematic alcohol use; brief intervention; training of health professionals; qualitative study; focal group.

\footnotetext{
${ }^{1}$ Psicóloga, pesquisadora do PAI-PAD/FMRP-USP

${ }^{2}$ Assistente Social, pesquisadora do PAI-PAD/FMRP-USP

${ }^{3}$ Médico Psiquiatra, pesquisador do PAI-PAD/FMRP-USP

${ }^{4}$ Professor Associado, Div. de Psicologia Médica, Membro da Comissão de Coord. do PAI-PAD/FMRP-USP

${ }^{5}$ Professor Doutor, Dep. de Medicina Social, Membro da Comissão de Coord. do PAI-PAD/FMRP-USP

${ }^{6}$ Professor Doutor, Div. de Psiquiatria, Coordenador Geral do PAI-PAD/FMRP-USP
}

\begin{tabular}{|c|c|c|c|c|}
\hline$S M A D 2005$ & $\begin{array}{l}\text { Volume } \\
\text { Volumen }\end{array}$ & $\begin{array}{l}\text { Número } \\
\text { Numero } \\
\text { Number }\end{array}$ & $\begin{array}{l}\text { Artigo } \\
\text { Artículo } 03 \\
\text { Article }\end{array}$ & http://www2.eerp.usp.br/resmad/artigos.asp \\
\hline
\end{tabular}




\section{INTRODUÇÃO}

Em meados dos anos setenta do século XX, cerca de trinta anos atrás, ocorreu a ampliação de conceitos e um melhor entendimento quanto aos problemas relacionados ao consumo de álcool. A dependência passou a ser entendida como apenas mais um dos problemas causados pelo uso excessivo de álcool, já que a maioria dos usuários não se tornava dependente, mas muitos sofriam outras conseqüências do uso ${ }^{(1)}$. Entre as conseqüências verificadas foram identificadas condições muito diversas como, por exemplo, acidentes automobilísticos, problemas sociais decorrentes do envolvimento com brigas ou desemprego, assim como problemas médicos em geral, entre outros.

A ampliação da visão em relação ao uso problemático de álcool permitiu que novos campos de estudos fossem abertos, com ênfase em prevenção, cuidados primários, planejamento de saúde, detecção e intervenção precoces ${ }^{(2)}$.

Visando a prevenção de problemas decorrentes do uso de álcool, na década de 80, a Organização Mundial de Saúde (OMS) desenvolveu um instrumento de rastreamento e propôs estratégias de intervenções breves para o uso abusivo de álcool ${ }^{(3-4)}$. Foi desenvolvido um instrumento denominado AUDIT (Alcohol Use Disorder Identification Test) e foram propostos níveis de intervenção, dependendo da pontuação do usuário no questionário. Essa forma de trabalho de reconhecimento precoce e intervenção rápida recebeu no Brasil o nome de EDIBs (Estratégias de Diagnóstico e Intervenções Breves) ${ }^{(2)}$.

A atenção primária à saúde (APS) foi escolhida como palco para o uso das EDIBs. Por ser o nível de atenção mais próximo à comunidade, os profissionais de APS - médicos, enfermeiras, assistentes sociais ou agentes comunitários de saúde - ocupam posição muito importante para identificar e intervir nos pacientes cujo padrão de beber está trazendo risco ou danos para a saúde ${ }^{(5)}$.

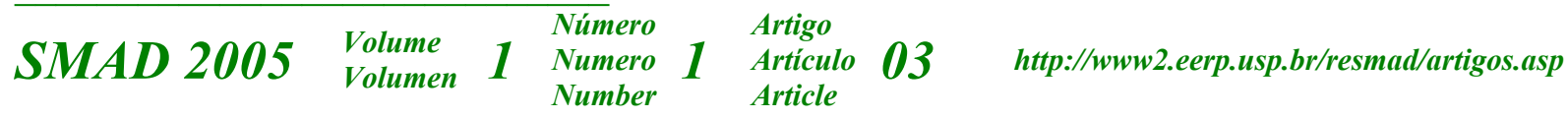


Em setembro de 2002, na cidade de Alicante, Espanha, em encontro promovido pela OMS, foi estabelecida uma parceria entre o Programa de Ações Integradas para Prevenção e Atenção ao Uso de Álcool e Drogas na Comunidade (PAI-PAD) da Faculdade de Medicina de Ribeirão Preto - USP e a OMS, visando a disseminação e implementação das EDIBs no Brasil. Com esse intuito, foi desenvolvido um programa de treinamento que, em acordo com a Direção Regional de Saúde de Ribeirão Preto (DIR-XVIII) da Secretaria Estadual de Saúde, e com prefeituras da região, vem sendo realizado periodicamente, atendendo prioritariamente as equipes do Programa de Saúde de Família e outros profissionais de saúde da rede pública. Inicialmente o treinamento foi dado aos profissionais de saúde de quatro núcleos do Programa de Saúde da Família (PSF), coordenados pelo Centro de Saúde-Escola (CSE) da Universidade de São Paulo (USP), Campus de Ribeirão Preto - SP. Desde então, equipes do PSF das cidades de Ribeirão Preto, Batatais e Cássia dos Coqueiros receberam treinamento, que deverá se estender a outros municípios e outras regionais no Estado de São Paulo ${ }^{(2)}$. As equipes do PSF são formadas basicamente por médico, enfermeira, auxiliares de enfermagem e agentes comunitários de saúde. Em alguns casos, outros profissionais podem ser encontrados nas equipes como, por exemplo, dentistas e psicólogos.

O treinamento oferecido teve como principal objetivo a capacitação dos profissionais das equipes do PSF para o emprego das EDIBs, tendo como eixo central de referência os manuais da OMS, do AUDIT e de Intervenções Breves, na versão do PAI-PAD, traduzida e adaptada ao português.

Os conteúdos abordados no treinamento foram: uso excessivo de álcool como problema de saúde pública, papel dos profissionais de saúde na prevenção de problemas relacionados ao uso de álcool, conceitos em relação ao uso de álcool (dose padrão, beber de baixo risco), manejo e interpretação do instrumento AUDIT e intervenções breves (Educação para o Álcool, Orientações Básicas, Aconselhamento Breve e Encaminhamento).

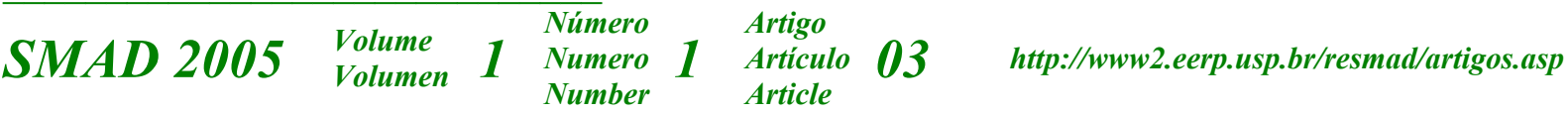


A fim de avaliar o treinamento oferecido, foi utilizada a estratégia de Grupo Focal. Tal estratégia foi escolhida por ser um método de pesquisa qualitativa que possibilita o contato com os diversos valores, crenças, hábitos, atitudes e percepções das pessoas sobre suas práticas, sobre um tema ou um produto. A riqueza dessa técnica advém do fato de captar a natureza dinâmica da interação do grupo, onde há espaço para concordância ou divergência a respeito das colocações e onde se podem observar tendências grupais. Essa estratégia já foi utilizada em outros estudos para avaliação da implementação de EDIBS e mostrou-se útil para esclarecer mudanças ocorridas no trabalho e dificuldades encontradas por profissionais de saúde na implementação de novos serviços ${ }^{(6-7)}$.

Considerando o acima exposto, o trabalho aqui relatado teve como objetivo investigar e avaliar os benefícios advindos do treinamento em EDIBs, do ponto de vista dos profissionais que receberam essa capacitação, com o uso de técnica de grupo focal.

\section{MÉTODOS}

Após aproximadamente quatro meses da realização do treinamento, a equipe do PAIPAD entrou em contato com as equipes dos Núcleos de Saúde da Família, propondo um encontro para a realização de Grupos Focais, com o objetivo de avaliar o treinamento e a implementação das EDIBS. Cada Núcleo ofereceu uma hora para que essa atividade fosse realizada e, ao final de dois meses, foram realizados quatro grupos focais, um em cada núcleo. Cada grupo teve a participação de seis a oito profissionais da equipe do PSF, que haviam recebido o treinamento do PAI-PAD. A proporção dos participantes do grupo refletiu a distribuição observada durante o treinamento, cuja maioria apresentava as seguintes características: cerca de $60 \%$ exercendo a função de agente comunitário de saúde, $85 \%$ do sexo feminino e $51 \%$ pertencente à faixa etária de adulto jovem, abaixo de 35 anos. 
A pauta foi dividida em dois temas: o primeiro era relacionado ao treinamento em si e aos conteúdos abordados por esse, e o segundo era relacionado à implementação das EDIBs.

Dada a natureza do estudo e em conformidade com a técnica de grupo focal, a seleção da amostra foi proposital. Foram convidados para participar dos grupos todos os profissionais que receberam o treinamento em EDIBs. Os participantes foram divididos em 4 grupos, de acordo com o Núcleo em que trabalhavam. Desta forma, foram realizados quatro grupos focais, um em cada NSF.

Cada grupo foi conduzido por um moderador e contou com a participação de dois observadores adicionais. Os grupos foram realizados em salas próprias de cada NSF, em horário cedido pelos profissionais dos núcleos. Cada grupo teve em torno de uma hora de duração. Além das anotações dos observadores, os depoimentos foram gravados em fitas magnéticas de áudio e depois transcritos na íntegra. As transcrições foram lidas e discutidas por três avaliadores. Com base nos objetivos da pesquisa, selecionou-se a primeira transcrição a ser analisada e cada leitor sublinhou todas as falas que se referiam aos benefícios conseqüentes do treinamento. Primeiramente foram discutidas as categorias encontradas e então se seguiu a leitura da segunda transcrição, do próximo grupo. O mesmo foi feito para a terceira e quarta transcrições. Com isso, definiram-se categorias de análise. Conhecendo-se as categorias, agruparam-se todas as falas relativas a cada uma delas para que fosse possível ter uma visão de possíveis subcategorias. Realizou-se então análise descritiva do tema "benefícios do treinamento". 


\section{RESULTADOS}

\section{Uso problemático de álcool}

Os profissionais apontaram como um dos benefícios do treinamento a mudança de visão que tinham em relação ao uso problemático de álcool. Relatam que antes do treinamento identificavam casos de dependência grave, quando o portador da síndrome já estava com várias áreas de sua vida prejudicadas e sua condição era visível a todos. Relataram que após o treinamento a visão do uso problemático de álcool se expandiu. Em relação à dependência de álcool, passaram a perceber que mesmo pessoas que trabalham e têm família podem estar dependentes. Algumas pessoas falaram sobre diferentes graus de dependência e apontaram a necessidade de maior aproximação para conseguirem identificar se o paciente está dependente de álcool ou não.

"O conceito do alcoólatra, bem diferente do que a gente vê hoje, o alcoólatra mesmo era aquele que vivia caindo, que bebia até... hoje não, hoje a gente sabe que em determinada fase a pessoa já é dependente”

A dependência de álcool também deixa de ser algo do campo da moral e passa para o campo da saúde, começando a ser vista como doença.

"Gozado, eu nunca pensei que uma pessoa que bebesse, que é alcoólatra, por exemplo, que ela é doente, era uma pessoa sem-vergonha, que gosta de beber, mas aí eu passei a perceber que não é por aí."

Começam também a ficar mais atentos ao chamado "beber social". Afirmam que antes do treinamento quando perguntavam ao paciente sobre o consumo de álcool, contentavam-se com a resposta "ah, eu bebo socialmente", ou, "tomo uma cervejinha no final de semana". Ao 
conhecerem as diferentes zonas de risco, perceberam a importância de investigarem o "beber social", pois entendem que pode colocar a pessoa em risco de desenvolver dependência.

"É a noção de que o indivíduo corre riscos de dependência ou não. É isto que eu quero dizer que antes a gente não tinha, que o treinamento nos deu. Porque você via uma pessoa, sabia até que ela usava álcool, mas você não tinha uma noção do risco que ela corria de estar dependente do álcool."

\section{Conceitos e capacitação técnica}

Os profissionais disseram que uma das dificuldades que encontravam para realizar uma intervenção sobre o uso problemático de álcool era a falta de conhecimento sobre o assunto. Após terem participado do treinamento, disseram ter aprendido como orientar em relação aos limites de consumo de baixo risco, a quantificar a dose-padrão, conheceram as situações em que não se deve beber, ampliaram os conhecimentos que tinham em relação às conseqüências do uso problemático de álcool, entenderam a importância de identificarem o estágio de prontidão para mudança em que o paciente se encontra e aprenderam estratégias motivacionais (por exemplo, "plantar a dúvida"). Esses conhecimentos, somados ao uso do questionário AUDIT para identificação de uso problemático de álcool, deu aos profissionais referência para a identificação do uso problemático e para a intervenção.

"Eu acho que a gente muda um pouco o jeito de escutar né, a história do 'ah, você usa bebida de álcool? Eu uso. Quanto? Tomo uma cervejinha de final de semana'. Essa coisa que a gente começou a escutar de uma maneira diferente, porque 'tomá' uma cervejinha de final de semana eu também tomo, né, aí que a gente começou a aprender a mexer com a história das doses, quantas doses, por quê que toma, e quantas vezes toma na semana, porque as pessoas, por exemplo, achavam que beber socialmente era tomar duas cervejas por dia e a gente vê isso e a gente então aprendeu agora a dar essa cutucada e a perguntar quanto que é beber socialmente 'ah, eu bebo socialmente. Mas então quanto é?, quantas que você bebe?, que tipo de bebida você usa?, quantas doses da bebida você toma?', então a gente aprendeu a olhar, a escutar isto de outra maneira porque é aquela história 'bebo de vez em quando', tudo bem, você anota lá: bebe socialmente, e com o AUDIT, com o treinamento a gente aprendeu a fazer isso de outra maneira, a gente agora sabe...".

SMAD $2005 \quad \begin{aligned} & \text { Volume } \\ & \text { Volumen }\end{aligned} 1 \begin{aligned} & \text { Número } \\ & \text { Numero } \\ & \text { Number }\end{aligned} 1 \begin{aligned} & \text { Artigo } \\ & \text { Artículo } \\ & \text { Article }\end{aligned} 03 \quad$ http://www2.eerp.usp.br/resmad/artigos.asp 
A aprendizagem desses conceitos novos foi percebida também como útil para o trabalho preventivo, sendo identificada então, como vindo ao encontro da função da atenção primária, que seria a de promoção e prevenção.

"E hoje a gente já tem esta visão e a gente já pode trabalhar com promoção que é o que nós temos que fazer, né? Eu acho que o treinamento deu mesmo esta noção, que foi bom para isto, porque os dependentes já são visíveis, a gente já sabe que são dependentes e que precisa tratar, né? Os familiares já sabem, os médicos já sabem. Mas as pessoas que estão em risco, que correm risco, a gente até sabia que podia ficar dependente, mas não tinha um instrumento para provar e mostrar isto pra própria pessoa."

\section{Abordagem}

Os profissionais identificaram como um dos benefícios do treinamento o fato de se sentirem mais preparados para abordar o assunto do uso problemático de álcool. Os conhecimentos que foram adquiridos possibilitaram que se sentissem mais seguros para conversarem sobre o assunto com seus pacientes e também para que entendessem melhor o processo de mudança, tornando a abordagem menos invasiva, baixando expectativas dos profissionais e aumentando o respeito pelo paciente.

"Falar disto, não teria coragem, como abordar. Não sabia como abordar, quando vi o homem da pinga, conversei com ele. Não sei nem falar de que jeito é, foi do meu jeito. Acho que ficou mais fácil da gente se colocar”.

\section{DISCUSSÃO}

O treinamento em intervenções breves para o uso problemático de álcool, oferecido pelo PAI-PAD estabeleceu objetivos cognitivos e também atitudinais. Ao se investigar os

\begin{tabular}{|c|c|c|c|c|}
\hline$S M A D 2005$ & $\begin{array}{l}\text { Volume } \\
\text { Volumen }\end{array}$ & $\begin{array}{l}\text { Número } \\
\text { Numero } \\
\text { Number }\end{array}$ & $\begin{array}{l}\begin{array}{l}\text { Artigo } \\
\text { Articulo } \\
\text { Article }\end{array} \\
\end{array}$ & http://www2.eerp.usp.br/resmad/artigos.asp \\
\hline
\end{tabular}


benefícios percebidos pelos profissionais treinados, foram identificados benefícios relacionados aos conhecimentos adquiridos e também a mudanças de atitude.

Através de conhecimentos sobre o que é a síndrome de dependência de álcool e o que são as zonas de risco, relacionadas com o consumo de álcool, foi possível questionar os estigmas relacionados aos estereótipos do "alcoólatra" e do "bêbado de sarjeta", tão freqüentes no imaginário popular, permitindo chamar a atenção para a necessidade da investigação regular e sistemática do uso problemático de álcool, que exige um olhar mais geral e mais aguçado. Com isso, a dependência de álcool também passou a ser vista como condição médica, dentro da categoria de doença/enfermidade, rompendo com o estigma do alcoolista "fraco", "sem caráter", ou de "pouca moral". Conclui-se que romper com o estereótipo e com o estigma ajuda o profissional a ampliar a investigação do uso problemático de álcool, assim como a entender melhor o contexto mais geral em que se localiza o padrão atual de consumo de álcool do paciente. Ao pensar a dependência como um problema de saúde, o usuário problemático ou dependente de álcool transita e se legitima dentro do âmbito das responsabilidades do profissional de saúde.

Ao conhecer as diferentes zonas de risco, o profissional passa a ficar mais atento ao "beber social", investigando o que ele significa e agindo de forma preventiva em seu trabalho. Com os conhecimentos sobre o beber de baixo risco, estágios de prontidão para mudança, estratégias motivacionais e conseqüências do uso de álcool torna-se possível oferecer intervenções mais individualizadas, centradas na problemática da pessoa que foi identificada como fazendo uso de risco, possibilitando intervenções e práticas preventivas e facilitando a abordagem do paciente.

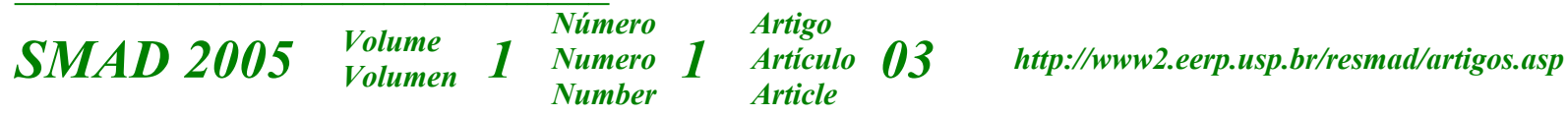




\section{REFERÊNCIAS BIBLIOGRÁFICAS}

1. Edwards G, Gross M. Alcohol dependence: provisional description of a clinical syndrome. Br Med J 1976; 1: 1058-61.

2. Furtado EF. Implementação de estratégias de diagnóstico e intervenções breves para problemas relacionados ao álcool em serviços de atenção primária na região de Ribeirão Preto. Ribeirão Preto: PAI-PAD/FMRP-USP; 2003. Relatório Técnico PAI-PAD/OMSBrazil-rp-01/2003.

3. Babor TF, Higgins-Biddle JC, Saunders JB, Monteiro MG. AUDIT: teste para identificação de problemas relacionados ao uso de álcool: roteiro para uso em atenção primária. Ribeirão Preto: PAI-PAD; 2003.

4. Babor TF, Higgins-Biddle JC. Intervenções breves para uso de risco e uso nocivo de álcool: manual para uso em atenção primária. Ribeirão Preto: PAI-PAD; 2003.

5. Marques ACPR, Furtado EF. Intervenções breves para problemas relacionados ao álcool. Rev Bras Psiquiatr 2004; 26 supl 1: 28-32.

6. Aalto M, Pekuri P, Seppa K. Obstacles to carrying out brief intervention for heavy drinkers in primary health care: a focus group study. Drug Alcohol Rev 2003; 22: 169-73.

7. Rush BR, Powell LY, Crowe TG, Ellis K. Early interventions for alcohol use: family physicians' motivations and perceived barriers. CMAJ 1995; 152: 863-9. 